\title{
Knowledge gaps in the appendix: a multi-institutional study from seven academic centers
}

\author{
Christina A. Arnold ${ }^{1} \cdot$ Rondell P. Graham $\mathbb{1}^{2} \cdot$ Dhanpat Jain $^{3} \cdot$ Sanjay Kakar ${ }^{4}$ Dora M. Lam-Himlin ${ }^{5}$ Bita V. Naini ${ }^{6}$. \\ Tsung-Teh $\mathrm{Wu}^{2} \cdot$ Matthew M. Yeh ${ }^{7} \cdot$ Michael S. Torbenson $^{2}$
}

Received: 24 October 2018 / Revised: 12 January 2019 / Accepted: 12 January 2019 / Published online: 14 February 2019

(c) United States \& Canadian Academy of Pathology 2019

\begin{abstract}
Appendix pathology represents uncommonly encountered specimens with unique diagnostic challenges. To delineate common knowledge gaps, extramural consults submitted to seven institutions between 2016-2017 were reviewed. All appendix consults were resections $(100 \%, n=43)$, and the majority were directed for consultation by the originating pathologist $(95 \%, n=41)$ with no additional studies performed by the consultant $(65 \%, n=28)$. This study was dominated by inquiries related to low grade appendiceal mucinous neoplasms $(44 \%, n=19)$ and goblet cell carcinoid related neoplasms $(19 \%, n=8)$. Of the 43 appendiceal consults, 19 were submitted by the contributing pathologist as low grade appendiceal mucinous neoplasm, but only half of these were diagnosed by the consultant as such $(n=9)$. Low grade appendiceal mucinous neoplasm-related consultation themes included diverticular disease, criteria for invasion, high grade atypia, extraappendiceal mucin, and staging. Examples of major disagreements that were downgraded included consults submitted as low grade appendiceal mucinous neoplasm and diagnosed by the consultant as serrated polyp $(n=3)$, appendicitis $(n=1)$, and benign appendix $(n=1)$. Examples of major disagreements-upgraded included cases submitted as low grade appendiceal mucinous neoplasm and diagnosed by the consultant as low grade appendiceal mucinous neoplasm with highrisk features $(n=2)$ and mucinous adenocarcinoma $(n=2)$. One case contained both a major disagreement-upgrade (low grade appendiceal mucinous neoplasm changed to high grade appendiceal mucinous neoplasm) and a major disagreementdowngrade (pT3 changed to Tis). Of the 15 cases diagnosed by the consultants as low grade appendiceal mucinous neoplasm, submitted diagnoses included low grade appendiceal mucinous neoplasm $(n=9)$, adenocarcinoma $(n=5)$, and one case was submitted without a diagnosis. For goblet cell carcinoid-related consults, the usual inquiry related to distinguishing goblet cell carcinoid from goblet cell carcinoid with adenocarcinoma (adenocarcinoma ex-goblet cell carcinoid). Of the 38 overall consults with a submitted diagnosis, $53 \%(n=20)$ were disagreements, and most of these were major disagreements-downgraded $(n=13)$.
\end{abstract}

\section{Introduction}

Pathology consultation cases can be classified as either extramural or referral-based. Extramural consults are cases

Christina A. Arnold

Christina.Arnold@OSUMC.edu

1 Department of Pathology, The Ohio State University Wexner Medical Center, Columbus, OH, USA

2 Department of Laboratory Medicine and Pathology, Mayo Clinic, Rochester, MN, USA

3 Department of Pathology, Yale School of Medicine, New Haven, CT, USA sent for expert review, usually at the direction of the originating pathologist before the diagnosis is finalized (also termed "primary consults"). In contrast, referral-based consults are reviewed after a diagnosis at an outside

4 Department of Pathology, University of California, San Francisco, San Francisco, CA, USA

5 Department of Laboratory Medicine and Pathology, Mayo Clinic, Scottsdale, AZ, USA

6 Department of Pathology, University of California, Los Angeles, Los Angeles, CA, USA

7 Department of Pathology, University of Washington School of Medicine, Seattle, WA, USA 
facility has been rendered at the request of the treating institution prior to treatment (also termed "secondary consults"). Both consultation types represent resource-rich materials that allow insights into important diagnostic issues. The largest study of referral-based consultations was performed by Mayo Clinic Rochester in 2013. Investigators studied more than 70,000 consults, identified an overall disagreement rate of $0.6 \%$, and reported that the most frequent area of disagreements was in gastrointestinal cases $(17.5 \%)$ [1]. While their overall disagreement rate was low, disagreements resulted in changes to treatment in $90 \%$ and prognosis in $92.1 \%$ of cases. Other consultation studies have reported higher disagreement rates in the range of $1.4-10 \%$ [2-6], with similar high impact on treatment and prognosis. This study evaluated extramural consults of the appendix submitted to seven academic institutions between 2016-2017.

\section{Materials and methods}

A study of extramural consults of the tubular gastrointestinal tract, appendix, liver, and pancreatobiliary tree resulted in the collection of 1365 consecutive consults submitted to nine participants at seven academic centers between 2016-2017. The nine participants were all consultant pathologists and they recorded the following deidentified data for each consult: person directing the case for consultation (pathologist, clinician, or patient), consultation question(s), site, pertinent history (present or absent) / results of imaging studies (present or absent) / relevant laboratory results (present or absent), submitted diagnosis, ancillary studies performed, and consultant's final diagnosis. All results were forwarded to MST who aggregated the consults by organ system: liver $40 \%(n=541)$, upper gastrointestinal tract 29\% $(n=390)$, lower gastrointestinal tract $21 \%(n=284)$, appendix $3 \%(n=43)$, pancreatobiliary tree $7 \%(n=96)$, and miscellaneous $0.8 \%(n=11)$. CAA scored all appendix consults and MST confirmed the scoring per the scoring strategy outlined in Table 1.

\section{Results}

\section{Overview of consults}

All appendix consults were resections $(100 \%, n=43)$, and the majority were directed for consultation by the originating pathologist $(95 \%, n=41$; clinician-directed $=5 \%$, $n=2$ ). Additional studies were usually not performed by the consultant $(65 \%, n=28)$. When additional studies were performed, they were most often H\&E levels $(60 \%, n=9)$. Of 38 cases with submitted diagnoses, half (19/38) were major disagreements, and most of them (13/19) were downgraded (Fig. 1). Most consults were submitted for tumor classification. Invasion assessment and serrated polyp classification were also common consultation inquiries (Fig. 2). Overlooked diagnoses from the submitting pathologist included granulomata $(n=1)$ and endometriosis $(n=1)$. Outdated or incorrect terminology in the submitted diagnoses included the terms "carcinoid" instead of "welldifferentiated neuroendocrine tumor" $(n=1)$, "mucinous cystic neoplasm" $(n=3)$ or "cystadenoma" $(n=1)$ instead of "low grade appendiceal mucinous neoplasm", and "adenocarcinoid" instead of "goblet cell carcinoid with adenocarcinoma (adenocarcinoma ex-goblet cell carcinoid)" $(n=1)$. Of the two consults directed by clinicians, one was classified in agreement with the original pathologist's diagnosis of "adenocarcinoma ex-goblet cell carcinoid" and the other was a major disagreement-downgrade from the original diagnosis of "adenocarcinoma" to the consultant's diagnosis of "low grade appendiceal mucinous neoplasm".

Table 1 Scoring strategy

\begin{tabular}{ll}
\hline Category & Definition \\
\hline No submitted diagnosis & $\begin{array}{l}\text { Consult submitted without diagnosis } \\
\text { The submitted diagnosis and consultant's } \\
\text { Agree }\end{array}$ \\
& diagnosis were synonymous
\end{tabular}

Minor disagreement

Major disagreementupgraded

Major disagreementdowngraded
There was no potential for significant change to clinical management or histologic classification

The consultant's diagnosis could result in more aggressive management and / or histologic classification

The consultant's diagnosis could result in less aggressive management and / or histologic classification
Example

See definition

Submitted diagnosis: LAMN,?LAMN, rule out LAMN, suspicious for LAMN, please evaluate for LAMN, mucinous cystic neoplasm; Consultant diagnosis: Low-grade appendiceal mucinous neoplasm (LAMN)

Submitted diagnosis: Acute appendicitis; Consultant diagnosis: Granulomatous appendicitis

Submitted diagnosis: LAMN; Consultant diagnosis: Moderately differentiated mucinous adenocarcinoma

Submitted diagnosis: LAMN; Consultant diagnosis: Appendiceal diverticulum 


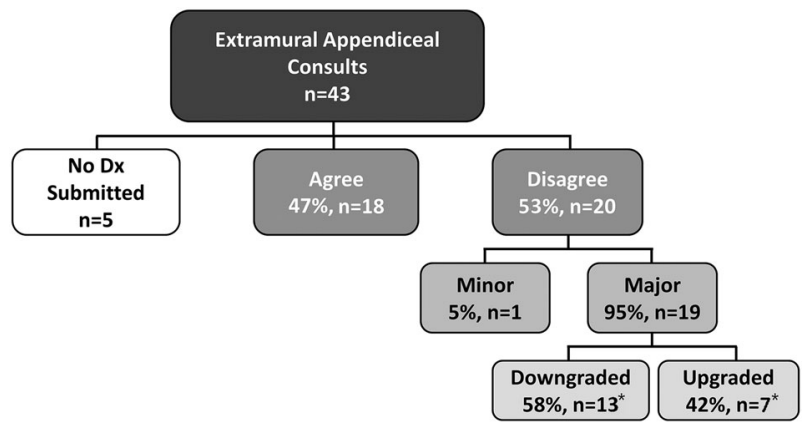

Fig. 1 Most study cases were finalized as major disagreements and downgraded from the submitted diagnosis. *One case had both a major disagreement-upgrade and downgrade: this case was submitted as low grade appendiceal mucinous neoplasm and upgraded by consultant to high grade appendiceal mucinous neoplasm (major disagreementupgraded) and a major disagreement-downgraded (submitted as T3 and downgraded by the consultant to Tis)

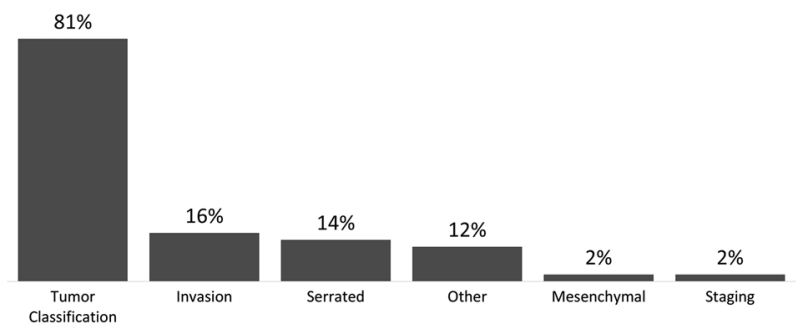

Fig. 2 The majority of consults were submitted for tumor classification. Invasion assessment and serrated lesion classification were also common concerns

\section{Low grade appendiceal mucinous neoplasm-related inquiries comprised the majority of consults}

Of the 43 total appendix consults, $44 \%(n=19)$ were submitted by the contributing pathologist as low grade appendiceal mucinous neoplasm, but only half of these were diagnosed by the consultant as low grade appendiceal mucinous neoplasm $(47 \%, n=9)$. Four consults submitted as low grade appendiceal mucinous neoplasm were scored as major disagreements-upgraded based on the consultant's diagnosis of low grade appendiceal mucinous neoplasm with high-risk features $(n=2)$ and moderately differentiated mucinous adenocarcinoma (G2) $(n=2)$. Five consults submitted as low grade appendiceal mucinous neoplasm were scored as major disagreements-downgraded based on the consultant's diagnosis of benign appendix $(n=1)$, appendicitis $(n=1)$, and serrated polyp $(n=3)$. One case had both a major disagreement-upgrade (submitted as a low grade appendiceal mucinous neoplasm and upgraded by consultant to high grade appendiceal mucinous neoplasm) and a major disagreement-downgrade (submitted as T3 and downgraded by the consultant to Tis). Next, we studied the submitting diagnoses for those consult cases diagnosed by the consultants as low grade appendiceal mucinous neoplasm $(n=15)$. Of these, the submitted diagnoses from the contributing pathologists included low grade appendiceal mucinous neoplasm $(n=9)$, adenocarcinoma $(n=5$, one of these was complicated by an appendiceal diverticulum), and one case was submitted without a diagnosis. Figures 3 and 4.

\section{Goblet cell carcinoid-related lesions were the second most common consult}

After low grade appendiceal mucinous neoplasm-related consults ( $49 \%$ of total consults, $n=21$ ), the next most common consultation theme was the classification of goblet cell carcinoid-related lesions ( $19 \%$ of total consults, $n=8$ ). The submitted diagnoses included the following: goblet cell carcinoid $(n=2)$, adenocarcinoma $(n=3)$, adenocarcinoid $(n=1)$, no submitted diagnosis $[n=2$; both diagnosed by the consultants as goblet cell carcinoid with adenocarcinoma (adenocarcinoma ex-goblet cell carcinoid)]. Of the cases with submitted diagnoses $(n=6)$, most were in agreement with the submitted diagnosis $(n=5)$. The one disagreement was a case submitted as "adenocarcinoma", but diagnosed by the consultant as "goblet cell carcinoid". Although goblet cell carcinoid and adenocarcinoma are staged and treated similarly, the change to histologic classification was regarded as significant and, consequently, scored as major disagreement-downgrade. Fig. 5.

\section{Discussion}

Major consultation themes of this series included tumor and serrated polyp classification, criteria for invasion, the significance of extra-appendiceal mucin, and staging, particularly as these diagnostic issues related to low grade appendiceal mucinous neoplasms. Fig. 2.

Regarding tumor classification, a common diagnostic issue was distinguishing a low grade appendiceal mucinous neoplasm from a serrated polyp. This distinction was particularly challenging in low grade appendiceal mucinous neoplasms with a normal caliber appendix not yet entirely overrun by the low grade appendiceal mucinous neoplasm, or, low grade appendiceal mucinous neoplasms with a villiform surface. As reported by others, gross features favoring a diagnosis of low grade appendiceal mucinous neoplasm included a distended appendix with abundant luminal mucin and a thinned bowel wall [7-11], whereas, appendices involved by serrated polyps were usually of normal caliber thickness. The typical histologic features of a low grade appendiceal mucinous neoplasm seen at low power included an attenuated appendiceal wall, lamina propria obliteration, a pushing border, and mucin dissection of the fibrotic wall [7-16]. On intermediate power, low 

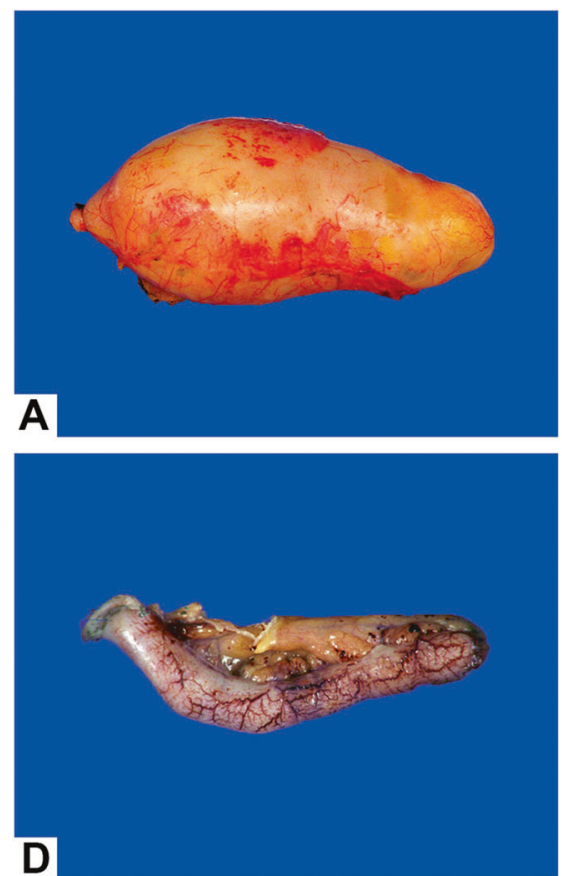

B
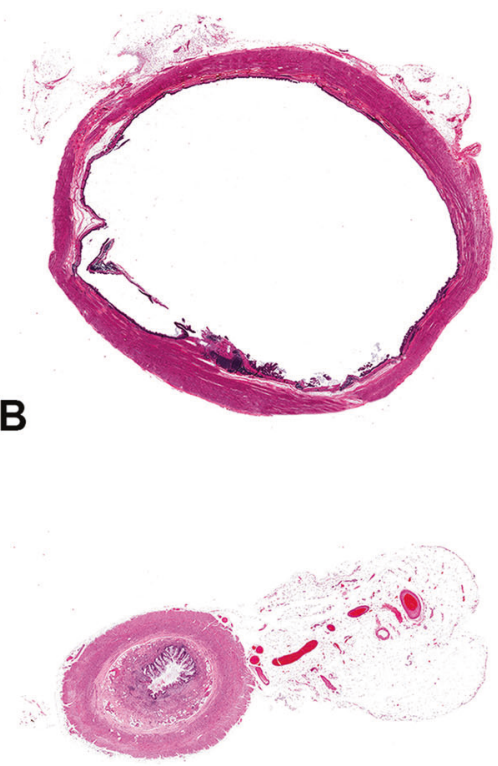

E
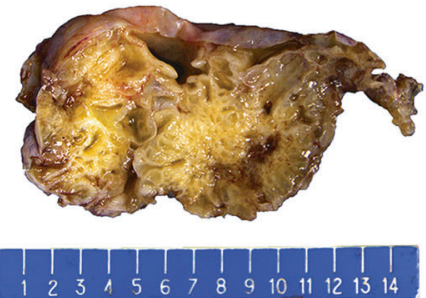

G

Fig. 3 Low grade appendiceal mucinous neoplasm versus serrated polyp versus adenocarcinoma was a common diagnostic issue. a Discriminating features of a low grade appendiceal mucinous neoplasm typically included a grossly distended appendix filled with abundant mucin. b, c This low grade appendiceal mucinous neoplasm was characterized by a thin wall (b), lamina propria obliteration (c), fibrosis of the submucosa and muscularis propria (c), and low-grade dysplasia of the mucinous epithelium (c). This case was submitted as possible low grade appendiceal mucinous neoplasm, diagnosed by the consultant as low grade appendiceal mucinous neoplasm, and classified as an agreement. d-f In contrast, an appendix involved by a serrated polyp showed normal caliber bowel wall thickness (d, e) and readily identifiable three bowel wall layers on low power (e). Intermediate power showed a preserved lamina propria, non-fibrotic submucosa (f), and serrated epithelium with easily identifiable goblet cells
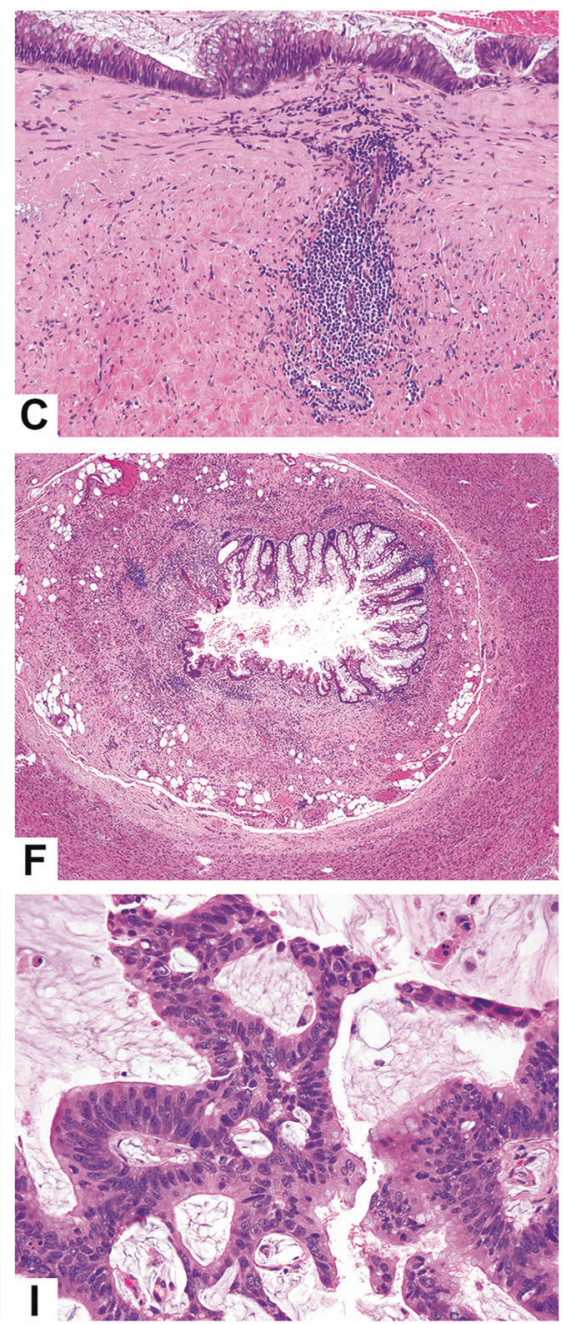

(f). This case was submitted as possible low grade appendiceal mucinous neoplasm, diagnosed by the consultant as serrated polyp, and classified as a major disagreement-downgraded. g Moderatelydifferentiated mucinous adenocarcinoma of the appendix demonstrated a complex gross configuration with solid and cystic components, hemorrhage and necrosis, and no grossly identifiable appendix. h, i The mucinous backdrop comprised more than $50 \%$ of the overall lesion. High grade features were seen with increased cellularity, complex glandular architecture, necrosis, prominent nucleoli, and loss of nuclear polarity. Stromal invasion with desmoplasia and individual cell infiltration were also seen (not shown). Signet ring cells were not identified. This case was submitted as possible low grade appendiceal mucinous neoplasm, diagnosed by the consultant as moderatelydifferentiated mucinous adenocarcinoma (G2), and classified as a major disagreement-upgraded

grade appendiceal mucinous neoplasms showed circumferential involvement of the appendiceal lumen by undulating or flat mucinous epithelium with low grade dysplasia, similar to the hyperchromasia and nuclear pseudostratification seen in conventional tubular adenomas [7-16]. In contrast, serrated polyps usually arose in appendices with normal bowel wall thickness, showed retention of the lamina propria, lacked a fibrotic wall, and displayed serrated epithelium with ovoid nuclei, eosinophilic cytoplasm, and readily identifiable goblet cells. Fig. 3 .

The distinction between a low grade appendiceal mucinous neoplasm and adenocarcinoma represented a major consultation theme in this series. Overall, we identified that appendiceal diverticular disease and low grade appendiceal mucinous neoplasm's broad, pushing border often raised concerns for invasion. Features that the consultants relied 

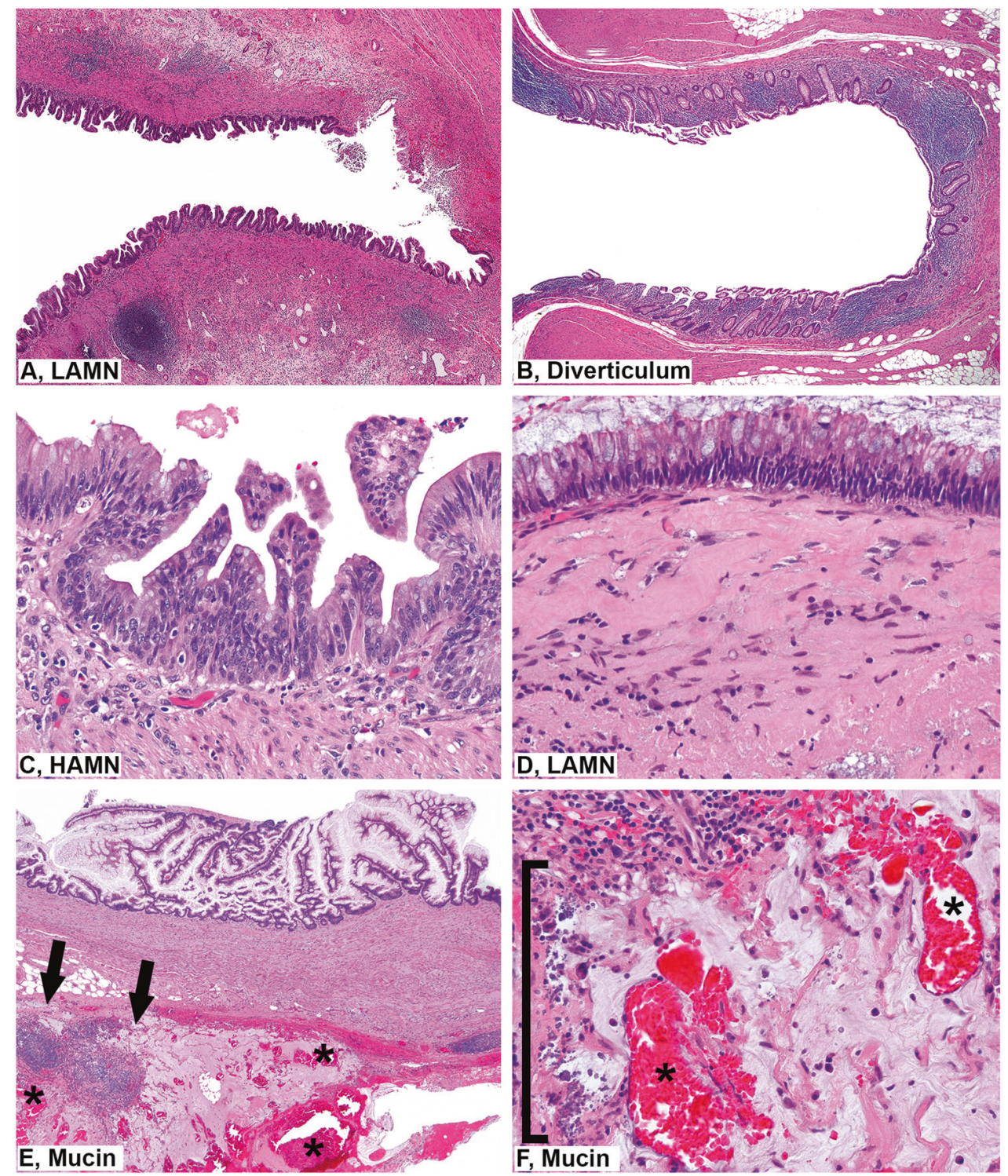

Fig. 4 Common low grade appendiceal mucinous neoplasm consultation themes. a, b Appendices involved by diverticular disease were diagnostic confounding issues. a Helpful diagnostic clues of a low grade appendiceal mucinous neoplasm included obliteration of the lamina propria and a fibrotic wall on low power, and low grade dysplasia of mucinous epithelium on intermediate power (not shown). b In contrast, diverticula retained the lamina propria and lacked dysplasia. Others have reported additional helpful features of diverticular disease include mucosal neuromas and epithelial disarray [36]. c A high grade appendiceal mucinous neoplasm was missed in one case. A high grade appendiceal mucinous neoplasm was defined as mucinous epithelium with all the architectural features of a low grade appendiceal mucinous neoplasm (not shown) and additionally high grade cytology (enlarged

on for definitive invasion included tumor budding, smallangulated glands, individual cell infiltration, desmoplasia, small nests of neoplastic glands suspended in mucin, and complex glandular growth [7, 14, 17]. More recently a "small cellular mucin pool" pattern has been described as a feature of infiltration whereby small mucin pools containing nuclei with loss of polarity, prominent nucleoli, and increased mitotic figures). This case was submitted as possible low grade appendiceal mucinous neoplasm, diagnosed by the consultant as high grade appendiceal mucinous neoplasm, and classified as a major disagreement-upgraded. d In contrast, a low grade appendiceal mucinous neoplasm is defined by low-grade cytology with retained nuclear polarity in mucinous epithelium. e, $\mathbf{f}$ The significance of extraappendiceal mucin was a common source of consultation. In contrast to mucin displaced by artifact of gross contamination, for example, real mucin featured tissue infiltration and a tissue reaction. On low power, the extra-appendiceal mucin infiltrated around lymphoid aggregates (e, arrows) and contained small capillaries (e, f, asterisks) and dystrophic calcifications (f, bracket)

neoplastic glands replace the appendiceal wall [11]. Other helpful features of invasion include the presence of signet ring cells, although one should be aware that epithelium suspended in mucin can simulate signet ring cells due to detachment and degeneration artifacts [18-23]. Helpful features of true signet rings include identification of the 


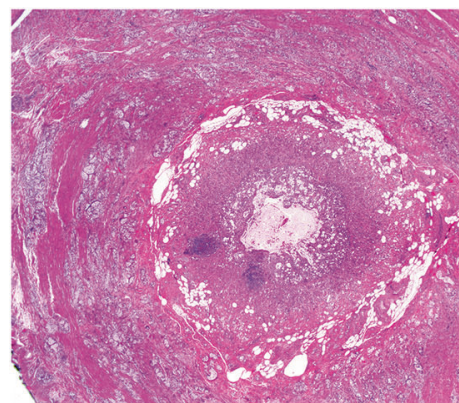

A

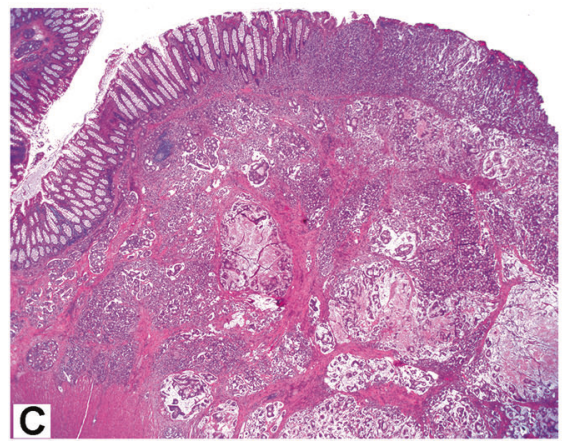

Fig. 5 After low grade appendiceal mucinous neoplasm-related consults, the second most common consult theme related to the classification of goblet cell neoplasms. a, b Under the Tang classification, this lesion was classified "typical goblet cell carcinoid" (group A) based on intact appendiceal architecture on low power (a), and well-defined goblet cell clusters lacking significant atypia and desmoplasia on high power (b) [28]. Under the Yozu classification, this same lesion was classified "low grade goblet cell adenocarcinoma" (grade 1) based on $>75 \%$ clustered growth pattern. c-d Under the Tang classification, this

signet rings in viable tissue with tissue infiltration and a desmoplastic reaction [18]. Davison et al suggest that the signet ring component reach a minimum threshold criteria of $>10 \%$ of the neoplasm for diagnosis [17], although this threshold is not required by the current American Joint Committee on Cancer (AJCC), 8th edition [24]. Worrisome gross features for invasion include complex gross configurations with readily identifiable necrosis, rupture, and hemorrhage (Fig. 3).

The concept of "high grade appendiceal mucinous neoplasm" was a diagnostic issue in one consult submitted as low grade appendiceal mucinous neoplasm. The diagnostic term "high grade appendiceal mucinous neoplasm" refers to appendiceal cases with the typical architecture of low grade appendiceal mucinous neoplasm, but with high-grade atypia as defined by loss of nuclear polarity, glandular complexity, increased nuclear: cytoplasmic ratio, prominent nucleoli, and/or increased mitotic figures [14] (Fig. 4). Importantly, the current AJCC, 8th edition recommends staging high grade appendiceal mucinous neoplasms as invasive adenocarcinoma due to a higher risk of recurrence, worse prognosis, and higher association with extra-appendiceal mucin with neoplastic cells, relative to low grade appendiceal

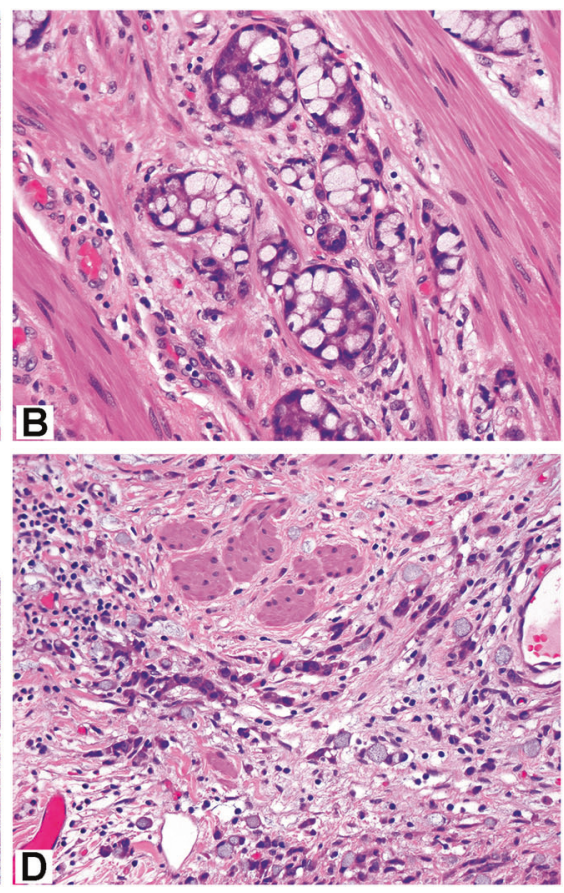

lesion was classified "adenocarcinoma ex-goblet cell carcinoid, signet ring cell type" (group B) based on destruction of the appendiceal wall on low power (c), and discohesive, single file infiltrative growth pattern, signet ring cells, and significant cytologic atypia seen on high power. This case lacked confluent growth. d Under the Yozu classification, this same lesion was classified as "high-grade goblet cell adenocarcinoma" (grade 3) based on the $<50 \%$ clustered growth pattern

mucinous neoplasms, in the small series available $[7,9,16$, 24, 25].

While there are no minimum criteria for extent of high grade atypia required to qualify for a diagnosis of high grade appendiceal mucinous neoplasm, one unequivocal focus, even if small, is sufficient for diagnosis per the Peritoneal Surface Oncology Group International [14]. By these standards, focal high grade atypia can be easy to miss in an entirely submitted, large appendix that commonly generates upwards of 40 glass slides. For practical purposes, the concept of high grade appendiceal mucinous neoplasm and when and how to report are controversial. In our centers, the finding of a single microscopic focus of high grade or questionable high grade component is reported as "predominantly low grade appendiceal mucinous neoplasm with focal areas of increased proliferation" $[11,17]$. The corresponding note documents the percentage of involvement of the high grade component and the lesion is staged as a low grade appendiceal mucinous neoplasm, assuming the high grade component comprises $<10 \%$ of the overall lesion. This is based on Davison et al.'s finding of no significant difference in survival between patients with low grade mucinous neoplasms and those with predominantly low 
grade tumors and $<10 \%$ of questionable microscopic foci of destructive invasion or nuclear atypia beyond that expected for low grade [17].

The classification of extra-appendiceal mucin in low grade appendiceal mucinous neoplasms was a major consultation theme. In the AJCC, 8th edition, a portion of the appendiceal staging is now based on the presence of mucin alone (without neoplastic cells) [24]. Preceding these changes, the terms "low grade appendiceal mucinous neoplasms with low-risk of recurrence" referred to low grade appendiceal mucinous neoplasms with extra-appendiceal mucin without neoplastic cells, and "low grade appendiceal mucinous neoplasms with high-risk of recurrence" referred to low grade appendiceal mucinous neoplasms with extraappendiceal mucin with neoplastic cells [10]. The new staging system underscores the importance of carefully evaluating the entirely submitted appendix and the importance of accurately distinguishing extra-appendiceal mucin from an artifact. Owing to the abundance of tenacious mucin within these lesions, it is not uncommon for the mucin on the external surface of the appendix to represent an artifact and originate from contamination via gross manipulation. Helpful tips arguing against an artifact include the identification of mucin dissecting into tissue and the presence of a tissue reaction, such as small capillary and granulation tissue in-growth, mesothelial hyperplasia, and dystrophic calcifications [11, 15] (Fig. 4).

Appendiceal nomenclature has evolved quickly in the past few years and was an issue addressed in this series. The submitted diagnoses of appendiceal "hyperplastic polyps" and "sessile serrated adenomas/polyps" were corrected to "serrated polyps" in accordance with the Peritoneal Surface Oncology Group International consensus statement [14]. This nomenclature change reflects that appendiceal serrated polyps are genetically disparate from their colonic counterparts, despite their identical morphology: appendiceal serrated polyps are characterized by KRAS mutations and colonic sessile serrated adenomas/polyps are more often characterized by BRAF mutations [26]. The outdated term "cystadenoma" (and "mucinous cystic neoplasm") was also corrected by the consultants to "low-grade appendiceal mucinous neoplasm" based on the Peritoneal Surface Oncology Group International consensus statement [14]. This shift in nomenclature clarifies the uncertain nature of this neoplasm and acknowledges its potential to cause concurrent or subsequent peritoneal disease, unlike a typical "adenoma" which is cured by simple excision.

After low grade appendiceal mucinous neoplasm-related consults, the classification of goblet cell neoplasms was the second most common consultation theme. Features contributing to these consults likely relate to their relatively uncommon incidence and evolving nomenclature and diagnostic criteria. In 1990, Burke et al proposed defining goblet cell carcinoid as goblet cell lesions with $<25 \%$ carcinomatous growth, and mixed carcinoid-adenocarcinoma as goblet cell lesions with $>50 \%$ carcinomatous growth [27]. In 2008, Tang et al. proposed an alternative classification: Typical goblet cell carcinoid (group A) referred to goblet cell lesions with well-defined goblet cell clusters lacking significant atypia, architectural distortion of the appendix, and desmoplasia; Adenocarcinoma ex goblet cell carcinoid, signet ring cell type (group B), referred to goblet cell lesions with goblet or signet ring cells, single cell infiltration, significant atypia, desmoplasia, destruction of the appendiceal wall, and lacking confluent growth; Adenocarcinoma ex-goblet cell carcinoid, poorly differentiated carcinoma (group C), referred to goblet cell lesions with at least focal goblet cells and $>1$ low power or $1 \mathrm{~mm}^{2}$ indistinguishable from a poorly-differentiated adenocarcinoma, which could appear as gland forming, confluent sheets, or undifferentiated carcinoma [28].

While Tang's updated system offered more granular histologic grading criteria, it sometimes has practical challenges in application. The lack of a minimum criterion for a signet ring component can be problematic. Classifying lesions with some, but not all, of the diagnostic criteria for a particular diagnostic category, and reliably discerning the distinction between goblet cells versus signet ring cells, and desmoplasia versus fibrosis present unique challenges. To address some of these challenges, in 2015, Lee et al proposed a simplified two-tier histologic grading system of low- versus high-grade goblet cell carcinoid based on cytologic atypia, stromal desmoplasia, and solid growth pattern [29]. More recently, in 2018 Yozu et al. proposed classifying all goblet cell carcinoid-related lesions as goblet cell adenocarcinoma with histologic grading of low, intermediate, and high grade based on the percentage of tubular or clustered growth [30]. In support of this classification, independent investigators demonstrated that goblet cell carcinoid and goblet cell carcinoid with adenocarcinoma (adenocarcinoma ex-goblet cell carcinoid) share similar mutational backgrounds and likely represent a morphologic spectrum of the same histologic entity, which is distinct from the usual neuroendocrine tumors and adenocarcinomas [31-33]. For practical purposes, providing both the Yozu classification to acknowledge the most recent molecular advances, along with the Tang classification, may serve as a prudent approach until this evolving issue is clarified (Fig. 5). Regardless of grading system employed, goblet cell carcinoid continues to be staged as adenocarcinoma in the AJCC, 8th edition [24].

Of note, Valasek et al recently reported their experience of 46 appendiceal referral-based consults collected between 2014-2015 [34]. Consults were identified through a computer-assisted search of appendiceal cases with primary mucinous neoplasm evaluated by outside pathologists, and 
then through the in-house slide review at the University of California San Diego over a 2-year period during patient referral for oncologic care. They similarly reported appendiceal knowledge gaps as the distinction between low grade appendiceal mucinous neoplasm and adenocarcinoma, recognition of high grade appendiceal mucinous neoplasm, outdated terminology, and incomplete reporting elements, such as documenting extra-appendiceal neoplastic epithelium. An overall disagreement rate of $28.3 \%$ was reported, and all disagreements were attributed to over-interpretation by the originating pathologist. Both our study and Valasak et al's identified higher disagreement rates than those previously reported in the range of $0.6-10 \%$ [1-6]. A possible explanation is that our studies focused exclusively on gastrointestinal-related cases, a field identified as the highest in disagreements according to the largest referral-consult study to date [1]. We suspect our overall 53\% disagreement rate was higher than Valasak et al.'s $28.3 \%$ disagreement rate based on the nature of the consults. None of the consults from the Valasak et al study were specifically requested by the originating pathologist (extramural based consults), whereas, our study was exclusively extramural based. Extramural consults are expected to have higher rates of disagreement because they are initiated by the diagnostic uncertainty of the originating pathologist.

The strengths of our study were also its weaknesses. The study incorporates more than 1300 extramural consults because multiple pathologists at multiple centers were involved. This allowed for an expansive study of common diagnostic themes across the country. However, it was not feasible for all nine pathologists at all seven sites to review all 1365 consults prospectively due to logistical constraints. By studying exclusively extramural consults, as opposed to referral-based consults, this study was enriched for diagnostic issues. However, since all cases were extramural consults with no readily accessible medical records for review by the consultants, it was not possible to record all clinical history, management, and outcomes, and, consequently, the designation of "major" versus "minor" discrepancies based on management distinctions was imperfect as it was theoretical and at the judgment of the authors. While the consultants were all gastrointestinalspecialized pathologists from major academic medical centers, the authors do not assume that the consultants were unilaterally correct in all cases, since many of the grey-zone areas were complicated by sometimes divergent criteria, subjective interpretation, and limited clinical context. Last, despite the broad study design, the 43 appendiceal consults collected represented a limited study size compared to all gastrointestinal, liver, and pancreatobiliary consults. Had the study been larger, we anticipate we may have encountered other challenging themes, such as endometriosis simulating low grade appendiceal mucinous neoplasm and appendiceal adenomas of the conventional type [35].

Despite these weaknesses, this study represented the largest study of gastrointestinal focused extramural consults, encompassing the broadest geographic territory of academic medical centers. We report a 53\% disagreement rate and identified common knowledge gaps in the appendix. These identified gaps can be leveraged to direct future academic studies, consensus guideline development, and improve educational opportunities.

Acknowledgements The authors would like to acknowledge Rick Marshall for assistance with the COPATH computer searches, Shawn Scully for assistance in image preparation, and Michael A. Arnold, $\mathrm{MD}, \mathrm{PhD}$ for salient discussions.

\section{Compliance with ethical standards}

Conflict of interest The authors declare that they have no conflict of interest.

Publisher's note: Springer Nature remains neutral with regard to jurisdictional claims in published maps and institutional affiliations.

\section{References}

1. Swapp RE, Aubry MC, Salomão DR, Cheville JC. Outside case review of surgical pathology for referred patients: the impact on patient care. Arch Pathol Lab Med. 2013;137:233-40.

2. Kronz JD, Westra WH, Epstein JI. Mandatory second opinion surgical pathology at a large referral hospital. Cancer. 1999;86:2426-35.

3. Manion E, Cohen MB, Weydert J. Mandatory second opinion in surgical pathology referral material: clinical consequences of major disagreements. Am J Surg Pathol. 2008;32:732-7.

4. Tsung JS. Institutional pathology consultation. Am J Surg Pathol. 2004;28:399-402.

5. Abt AB, Abt LG, Olt GJ. The effect of interinstitution anatomic pathology consultation on patient care. Arch Pathol Lab Med. 1995;119:514-7.

6. Renshaw AA, Gould EW. Reducing false-negative and falsepositive diagnoses in anatomic pathology consultation material. Arch Pathol Lab Med. 2013;137:1770-3.

7. Carr NJ, Bibeau F, Bradley RF, Dartigues P, Feakins RM, Geisinger KR, et al. The histopathological classification, diagnosis and differential diagnosis of mucinous appendiceal neoplasms, appendiceal adenocarcinomas and pseudomyxoma peritonei. Histopathology. 2017;71:847-58.

8. Misdraji J. Mucinous epithelial neoplasms of the appendix and pseudomyxoma peritonei. Mod Pathol. 2015;28(Suppl 1):S67-79.

9. Misdraji J, Yantiss RK, Graeme-Cook FM, Balis UJ, Young RH. Appendiceal mucinous neoplasms: a clinicopathologic analysis of 107 cases. Am J Surg Pathol. 2003;27:1089-103.

10. Pai RK, Beck AH, Norton JA, Longacre TA. Appendiceal mucinous neoplasms: clinicopathologic study of 116 cases with analysis of factors predicting recurrence. Am J Surg Pathol. 2009;33:1425-39.

11. Valasek MA, Pai RK. An update on the diagnosis, grading, and staging of appendiceal mucinous neoplasms. Adv Anat Pathol. 2018;25:38-60. 
12. Bellizzi AM, Rock J, Marsh WL, Frankel WL. Serrated lesions of the appendix: a morphologic and immunohistochemical appraisal. Am J Clin Pathol. 2010;133:623-32.

13. Yantiss RK, Panczykowski A, Misdraji J, Hahn HP, Odze RD, Rennert $\mathrm{H}$, et al. A comprehensive study of nondysplastic and dysplastic serrated polyps of the vermiform appendix. Am J Surg Pathol. 2007;31:1742-53.

14. Carr NJ, Cecil TD, Mohamed F, Sobin LH, Sugarbaker PH, Gonzalez-Moreno S, et al. A consensus for classification and pathologic reporting of pseudomyxoma peritonei and associated appendiceal neoplasia: the results of the Peritoneal Surface Oncology Group International (PSOGI) Modified Delphi Process. Am J Surg Pathol. 2016;40:14-26.

15. Umetsu SE, Shafizadeh N, Kakar S. Grading and staging mucinous neoplasms of the appendix: a case series and review of the literature. Hum Pathol. 2017;69:81-9.

16. Yantiss RK, Shia J, Klimstra DS, Hahn HP, Odze RD, Misdraji J. Prognostic significance of localized extra-appendiceal mucin deposition in appendiceal mucinous neoplasms. Am J Surg Pathol. 2009;33:248-55.

17. Davison JM, Choudry HA, Pingpank JF, Ahrendt SA, Holtzman MP, Zureikat AH, et al. Clinicopathologic and molecular analysis of disseminated appendiceal mucinous neoplasms: identification of factors predicting survival and proposed criteria for a three-tiered assessment of tumor grade. Mod Pathol. 2014;27:1521-39.

18. Sirintrapun SJ, Blackham AU, Russell G, Votanopoulos K, Stewart JH, Shen P, et al. Significance of signet ring cells in highgrade mucinous adenocarcinoma of the peritoneum from appendiceal origin. Hum Pathol. 2014;45:1597-604.

19. Boncher J, Bronner M, Goldblum JR, Liu X. Reticulin staining clarifies florid benign signet ring cell change with mitotic activity in a penetrating gastric ulcer. Am J Surg Pathol. 2011;35:762-6.

20. Ragazzi M, Carbonara C, Rosai J. Nonneoplastic signet-ring cells in the gallbladder and uterine cervix. A potential source of overdiagnosis. Hum Pathol. 2009;40:326-31.

21. Wang K, Weinrach D, Lal A, Musunuri S, Ramirez J, Ozer O, et al. Signet-ring cell change versus signet-ring cell carcinoma: a comparative analysis. Am J Surg Pathol. 2003;27:1429-33.

22. Suri VS, Sakhuja P, Malhotra V, Gondal R, Sachdev AK, Negi SS. Benign signet ring cell change with multilayering in the gallbladder mucosa — a case report. Pathol Res Pract. 2001;197:785-8.

23. Michal M, Chlumska A, Mukensnabl P. Signet-ring cell aggregates simulating carcinoma in colon and gallbladder mucosa. Pathol Res Pract. 1998;194:197-200.

24. Overman MJ, Asare EA, Compton CC, Hanna NH, Kakar S, Kosinski LA, et al. Appendix-carcinoma. NY, New York. 8th ed. Springer; 2017. pp. 237-50.
25. Carr NJ, Cecil TD, González-Moreno S, Mohamed F, Moran BJ, Sobin LH, et al. In reply. Am J Surg Pathol. 2016;40:1578.

26. Pai RK, Hartman DJ, Gonzalo DH, Lai KK, Downs-Kelly E, Goldblum JR, et al. Serrated lesions of the appendix frequently harbor KRAS mutations and not BRAF mutations indicating a distinctly different serrated neoplastic pathway in the appendix. Hum Pathol. 2014;45:227-35.

27. Burke AP, Sobin LH, Federspiel BH, Shekitka KM, Helwig EB. Goblet cell carcinoids and related tumors of the vermiform appendix. Am J Clin Pathol. 1990;94:27-35.

28. Tang LH, Shia J, Soslow RA, Dhall D, Wong WD, O'Reilly E, et al. Pathologic classification and clinical behavior of the spectrum of goblet cell carcinoid tumors of the appendix. Am J Surg Pathol. 2008;32:1429-43.

29. Lee LH, McConnell YJ, Tsang E, Zerhouni S, Speers C, Kennecke $\mathrm{H}$, et al. Simplified 2-tier histologic grading system accurately predicts outcomes in goblet cell carcinoid of the appendix. Hum Pathol. 2015;46:1881-9.

30. Yozu M, Johncilla ME, Srivastava A, Ryan DP, Cusack JC, Doyle L, et al. Histologic and outcome study supports reclassifying appendiceal goblet cell carcinoids as goblet cell adenocarcinomas, and grading and staging similarly to colonic adenocarcinomas. Am J Surg Pathol. 2018;42:898-910.

31. Johncilla M, Stachler M, Misdraji J, Lisovsky M, Yozu M, Lindeman N, et al. Mutational landscape of goblet cell carcinoids and adenocarcinoma ex goblet cell carcinoids of the appendix is distinct from typical carcinoids and colorectal adenocarcinomas. Mod Pathol. 2018;31:989-96.

32. Jesinghaus M, Konukiewitz B, Foersch S, Stenzinger A, Steiger $\mathrm{K}$, Muckenhuber A, et al. Appendiceal goblet cell carcinoids and adenocarcinomas ex-goblet cell carcinoid are genetically distinct from primary colorectal-type adenocarcinoma of the appendix. Mod Pathol. 2018;31:829-39.

33. Wen KW, Grenert JP, Joseph NM, Shafizadeh N, Huang A, Hosseini M, et al. Genomic profile of appendiceal goblet cell carcinoid is distinct compared to appendiceal neuroendocrine tumor and conventional adenocarcinoma. Hum Pathol. 2018;77:166-74.

34. Valasek MA, Thung I, Gollapalle E, Hodkoff AA, Kelly KJ, Baumgartner JM, et al. Overinterpretation is common in pathological diagnosis of appendix cancer during patient referral for oncologic care. PLoS ONE. 2017;12:e0179216.

35. Misdraji J, Lauwers GY, Irving JA, Batts KP, Young RH. Appendiceal or cecal endometriosis with intestinal metaplasia: a potential mimic of appendiceal mucinous neoplasms. Am J Surg Pathol. 2014;38:698-705.

36. Hsu M, Young RH, Misdraji J. Ruptured appendiceal diverticula mimicking low-grade appendiceal mucinous neoplasms. Am J Surg Pathol. 2009;33:1515-21. 